\title{
Differences between daytime and nighttime blood pressure variability regarding systemic atherosclerotic change and renal function
}

\author{
Tatsuo Kawai, Mitsuru Ohishi, Kei Kamide, Chikako Nakama, Miyuki Onishi, Norihisa Ito, Yoichi Takami, \\ Yasushi Takeya and Hiromi Rakugi
}

Recently, new parameters related to hypertension, such as variability in blood pressure and ambulatory arterial stiffness index (AASI), were demonstrated to correlate with arteriosclerotic change. In this study, we investigated the correlation between circadian variability in blood pressure/AASI and renal function. We also investigated differences in the clinical impact of $24 \mathrm{~h}$, daytime and nighttime blood pressure variability on renal and systemic atherosclerotic changes. We analyzed data from 120 patients who underwent renal Doppler ultrasonography (RDU) and ambulatory blood pressure monitoring (ABPM) at our hospital ward, and investigated the correlation between circadian variability in blood pressure/AASI and renal function, including resistive index (RI) evaluated with RDU, which is thought to be a good indicator of renal vascular resistance. Subjects with higher circadian variability in systolic blood pressure (SBP) had significantly higher RI. Daytime variability in SBP correlated more strongly with RI than nighttime variability. Meanwhile, only nighttime variability, but not daytime variability, in SBP was related to carotid atherosclerosis. Similarly, AASI was significantly correlated with RI. Circadian variability in SBP and AASI were both significantly correlated with renal function. Daytime SBP s.d. was especially more strongly correlated with renal vascular resistance, and nighttime SBP s.d. was significantly correlated with intima-media thickness (IMT) and plaque score. These results indicate that evaluating both daytime and nighttime blood pressure variability enables an assessment of pathological conditions in hypertensive patients to prevent cardiovascular diseases. Hypertension Research (2013) 36, 232-239; doi:10.1038/hr.2012.162; published online 18 October 2012

Keywords: AASI; arteriosclerosis; renal vascular resistance; resistive index; variability of blood pressure

\section{INTRODUCTION}

Much evidence establishes that hypertension involves an increased risk of cardiovascular disease, such as chronic kidney disease (CKD) ${ }^{1,2}$ To manage hypertensive patients, ambulatory blood pressure monitoring (ABPM) is widely recognized as a valuable examination, as previous studies reported that 24 -h blood pressure (BP) showed reproducible values and was more accurately correlated with cardiovascular diseases ${ }^{3,4}$ and prognosis ${ }^{5,6}$ than office BP.

Recently, in addition to 24-h BP, diurnal BP changes evaluated by ABPM, such as morning surge and nocturnal BP fall, was reported to correlate with cardiovascular disease and mortality. ${ }^{7-9}$ We have also previously reported that the morning BP surge is significantly correlated with renal vascular damage. ${ }^{10}$ Moreover, several studies showed that greater circadian variability (s.d.) in BP evaluated by $A B P M$ caused aggravated target organ damage ${ }^{11,12}$ or increased the ratio of cardiovascular events. ${ }^{13,14}$ However, as mentioned above, diurnal BP changes, such as nocturnal BP fall or rise, is also reported to correlate with cardiovascular events, and these diurnal BP changes affect the 24 -h variability; ${ }^{15}$ therefore, the clinical impact of 24 -h variability in $\mathrm{BP}$ might be disturbed. In fact, in a large population cohort study, Hansen et al. ${ }^{16}$ indicated that $24-\mathrm{h}$ BP variability did not contribute much to risk stratification over and beyond 24-h BP. Therefore, recent studies have investigated the clinical significance of daytime and nighttime variability and weighted variability that was not affected by nocturnal BP fall or rise, respectively. For example, daytime systolic blood pressure (SBP) variability was reported to correlate with target organ damage in hypertensive subjects, ${ }^{17}$ and nighttime BP variability was reported to be a strong predictor of cardiovascular events in patients with type 2 diabetes. ${ }^{18}$ However, there is still limited information about the correlation between circadian BP variability and renal atherosclerotic changes and the difference in clinical significance of the variability upon systemic atherosclerotic change.

Recently, Li et al. ${ }^{19,20}$ suggested a new index assessed from ABPM, ambulatory arterial stiffness index (AASI), which was a good indicator of arterial stiffness. They reported that AASI correlated 
well with other evaluations of arterial stiffness, such as pulse wave velocity and augmented index, and that AASI could provide prognostic information on cardiovascular mortality. Other studies showed that increased AASI is associated with target organ damage, decreased glomerular filtration ratio, or past history of stroke. ${ }^{21-23}$ However, no study has directly investigated the correlation between AASI and renal atherosclerotic change.

Resistive index (RI: (peak systolic velocity - end diastolic velocity)/ peak systolic velocity at segmental arteries in kidney) assessed by renal Doppler ultrasonography (RDU) is a valuable index of renal vascular resistance related to arteriosclerosis ${ }^{24-26}$ and provides good prognostic information regarding renal function. ${ }^{27-29}$ We also found that RI is a more efficacious index for evaluating very early renal damage than estimated glomerular filtration rate (eGFR). ${ }^{10}$ Therefore, we think that renal vascular damage caused by atherosclerotic risk factors, such as circadian BP variability or AASI, could be evaluated more precisely by using RI.

In this study, we assessed the hypothesis that the circadian variability in BP and AASI correlates significantly with renal atherosclerotic change. We investigated the correlation between circadian BP variability (expressed as s.d.) and various renal function parameters, such as eGFR, RI and level of proteinuria. Additionally, we investigated the difference in clinical impact upon the renal and systemic atherosclerotic changes in 24-h BP variability, and daytime and nighttime BP variability and AASI.

\section{METHODS}

\section{Study subjects and study design}

In our hospital ward, almost all patients admitted for several internal diseases, such as diabetes mellitus, hypertension, dyslipidemia, CKD and so on, undergo RDU to evaluate renal arteriosclerotic changes. We initially enrolled 281 consecutive patients, with and without CKD, undergoing RDU in our hospital ward between February 2009 and May 2011. Patients were excluded if they had renal artery stenosis $(n=16)$, renal transplant $(n=1)$, or were on dialysis $(n=1)$. Of the 263 patients, data from 120 patients undergoing ABPM to evaluate diurnal BP changes were analyzed in this study.

Subjects underwent biochemical examinations of blood and urine. Clinical parameters considered in this study included height, weight, body mass index (BMI), eGFR, serum lipid profile, fasting blood glucose, hemoglobin Alc (HbAlc), additional biochemical parameters, serum protein level, SBP and diastolic BP (DBP) at the time of RDU, smoking history and drug profile. Patients with diabetes mellitus were diagnosed according to the diagnostic criteria of the American Diabetes Association; fasting plasma glucose at or above $126 \mathrm{mg} \mathrm{dl}^{-1}, \mathrm{HbAlc} \geqslant 6.5 \%$, a 2 -h value in an oral glucose tolerance test at or above $200 \mathrm{mgdl}^{-1}$, or a random plasma glucose concentration $\geqslant 200 \mathrm{mg} \mathrm{dl}^{-1}$ in the presence of symptoms, or taking drugs for diabetes, and patients with hyperlipidemia were diagnosed as that; total cholesterol $\geqslant 220 \mathrm{mg} \mathrm{dl}^{-1}$. LDL cholesterol $\geqslant 140 \mathrm{mg} \mathrm{dl}^{-1}$, triglyceride $\geqslant 150 \mathrm{mg} \mathrm{dl}^{-1}$, or taking drugs for hyperlipidemia. The Clinical Investigations Ethics committee of Osaka University Hospital approved the study protocol and written informed consent was obtained from all participants. The study was performed in according to the ethical principles of the Declaration of Helsinki and Good Clinical Practice standards.

\section{Ultrasonographic determination}

RI was calculated as:

$\mathrm{RI}=($ peak systolic velocity - end diastolic velocity $) /$ peak systolic velocity.

Patients were placed in a supine position, and the size of the left and right kidneys and the flow velocity in the aorta and renal arteries were evaluated to detect morphological abnormalities or renal artery stenosis. RI was determined in three different segmental arteries in both kidneys, and expressed as the mean of these values. This method was reported to be identical and technically easy to perform such that the reproducibility of RI could be improved. ${ }^{30-32}$
Previous studies indicated that reliable RI measurements depended on proper measurement techniques that are performed by experienced operators. ${ }^{33}$ In this study, Doppler examinations were performed by three experienced operators (T.K., K.K. and M.O.) using a XARIO SSA-660A ultrasound machine (Toshiba, Tokyo, Japan) with a $2.5-\mathrm{MHz}$ sector transducer. Similarly, these specially trained technicians examined the echocardiograms and carotid ultrasonograms, and took the mean of three examinations as a representative measurement. Plaque score and the mean intima-media thickness (IMT) of the carotid artery were determined by carotid ultrasonography as previously reported. ${ }^{34}$

\section{Ambulatory blood pressure monitoring}

Ambulatory blood pressure was evaluated using portable monitors (FM-200, Fukuda Denshi, Tokyo, Japan) at 30-min intervals throughout the entire day or at 30-min intervals during the daytime (0700 hours to 2200 hours) and 60-min intervals at night ( 2200 hours to 0700 hours). In the present study, our subjects underwent ABPM in hospitalized condition. We defined circadian variability in BP as the s.d. in SBP, and we analyzed all-day SBP s.d., daytime (0700 hours to 2200 hours) SBP s.d. and nighttime (2200 hours to 0700 hours) SBP s.d. From SBP values obtained from ABPM, AASI was calculated as follows:

$$
1 \text {-regression slope of DBP on SBP. }{ }^{19}
$$

\section{Renal function}

eGFR was calculated using the following equation: ${ }^{35}$

$$
\begin{aligned}
& \text { eGFR }\left(\mathrm{ml} \mathrm{min}^{-1} \text { per } 1.73 \mathrm{~m}^{2}\right)=194 \times\left(\text { creatinine }^{-1.094}\right) \\
& \times\left(\text { Age }^{-0.287}\right)(\times 0.739 \text { if female }) .
\end{aligned}
$$

The level of albuminuria was evaluated according to the American Diabetes Association classification. ${ }^{36}$ The albumin/creatinine ratio in spot urine was used to classify proteinuria as follows: no proteinuria, $<30 \mathrm{mg} \mathrm{g}^{-1}$ creatinine; microalbuminuria, $30-300 \mathrm{mg} \mathrm{g}^{-1}$ creatinine; clinical albuminuria, $\geqslant 300 \mathrm{mg} \mathrm{g}^{-1}$ creatinine.

\section{Statistical analysis}

Data were analyzed using JMP ver. 9.0.1 (SAS, Cary, NC, USA), and are presented as the mean \pm s.e.m. Differences between groups were analyzed employing the unpaired Student's $t$-test and Pearson's chi-square test. Multiple regression analysis was used to identify possible determinants of RI. A value of $P<0.05$ was regarded as significant.

\section{RESULTS}

Table 1 reports the baseline clinical characteristics and usage of antihypertensive agents of the patients. The mean patient age was $68.8 \pm 1.1$ years.

First, we compared the characteristics between subjects with greater SBP s.d. and subjects with lower SBP s.d. (Table 2). In the all-day variability, subjects with greater SBP s.d. were significantly older, and had the morbidities of dyslipidemia and elevated HbAlc. There was also a significantly lower male-to-female ratio. In addition, subjects with greater SBP s.d. had significantly higher mean IMT and plaques as evaluated by carotid Doppler ultrasonography, and RI as evaluated by RDU. In daytime variability, subjects with greater SBP s.d. were significantly older, had higher BMI, the morbidities of diabetes mellitus, dyslipidemia and elevated $\mathrm{HbAlc}$, and, moreover, significantly higher RI. In nighttime variability, subjects with greater SBP s.d. were significantly older, and had higher mean IMT, plaque score and RI. Similarly, Table 3 shows the comparison of baseline characteristics and parameters related to systemic atherosclerotic changes between higher and lower AASI. Subjects with higher AASI had significantly higher BMI and RI. There were no significant differences in the usage of respective class of antihypertensive agents 
Table 1 Baseline clinical characteristics of the study population

\begin{tabular}{|c|c|}
\hline & Total \\
\hline Number & 120 \\
\hline Sex (male/female) & $50 / 70$ \\
\hline Age (years) & $68.8 \pm 1.1$ \\
\hline BMI $\left(\mathrm{kg} \mathrm{m}^{-2}\right)$ & $23.8 \pm 0.4$ \\
\hline Diabetes mellitus $(n, \%)$ & $33(30.8 \%)$ \\
\hline Dyslipidemia $(n, \%)$ & $43(36.1 \%)$ \\
\hline eGFR $\left(\mathrm{ml} \mathrm{min}{ }^{-1}\right.$ per $\left.1.73 \mathrm{~m}^{2}\right)$ & $61.3 \pm 2.0$ \\
\hline 24-h SBP (mm Hg) & $132.0 \pm 1.6$ \\
\hline 24-h DBP (mm Hg) & $77.8 \pm 1.1$ \\
\hline Total cholesterol ( $\mathrm{mg} \mathrm{dl}^{-1}$ ) & $194.2 \pm 3.2$ \\
\hline Triglyceride $\left(\mathrm{mg} \mathrm{dl}^{-1}\right)$ & $124.1 \pm 6.5$ \\
\hline HDL cholesterol (mg dl $\left.{ }^{-1}\right)$ & $55.7 \pm 1.6$ \\
\hline LDL cholesterol (mg dl ${ }^{-1}$ ) & $112.9 \pm 2.8$ \\
\hline $\mathrm{UA}\left(\mathrm{mg} \mathrm{dl}^{-1}\right)$ & $5.9 \pm 0.1$ \\
\hline HbAlc (\%) & $5.8 \pm 1.1$ \\
\hline Antihypertensive agents ( $n, \%)$ & $61(92.5 \%)$ \\
\hline $\operatorname{ARB}(n, \%)$ & $61(50.8 \%)$ \\
\hline $\operatorname{ACEI}(n, \%)$ & 12 (10.0\%) \\
\hline $\operatorname{CCB}(n, \%)$ & $89(74.2 \%)$ \\
\hline Diuretic ( $n, \%)$ & $35(29.2 \%)$ \\
\hline Beta blocker ( $n, \%)$ & $27(22.5 \%)$ \\
\hline Alpha blocker $(n, \%)$ & $16(13.3 \%)$ \\
\hline Aldosterone blocker ( $n, \%)$ & $6(5.0 \%)$ \\
\hline
\end{tabular}

Values are expressed as mean \pm s.e.m. or numbers.

Abbreviations: ABPM, ambulatory blood pressure monitoring; ACEI, angiotensin-converting enzyme inhibitor; $\mathrm{ARB}$, angiotensin II receptor blocker; $\mathrm{BMI}$, body mass index; $\mathrm{CCB}$, calcium channel blocker; eGFR, glomerular filtration rate; HbAlc, hemoglobin Alc; HDL, high-density lipoprotein; LDL, low-density lipoprotein; UA, uric acid; 24-h DBP, 24-h diastolic blood pressure (all-day, evaluated with ABPM); 24-h SBP, 24-h systolic blood pressure (all-day, evaluated with ABPM).
Table 3 Comparison of baseline characteristics and paameters related to systemic atherosclerotic changes between subjects with higher AASI and subjects with lower AASI

\begin{tabular}{|c|c|c|c|}
\hline & \multicolumn{2}{|c|}{ AASI } & \multirow[b]{2}{*}{ P-value } \\
\hline & Higher & Lower & \\
\hline Number & 60 & 60 & \\
\hline Sex (male/female) & 23/37 & 27/33 & 0.4589 \\
\hline Age (years) & $68.5 \pm 1.6$ & $69.0 \pm 1.6$ & 0.5843 \\
\hline BMI (kg m-2) & $24.8 \pm 4.6$ & $22.8 \pm 3.2$ & 0.0028 \\
\hline Diabetes mellitus ( $n, \%)$ & 19 (35.9\%) & $14(25.9 \%)$ & 0.2665 \\
\hline Dyslipidemia $(n, \%)$ & $22(36.7 \%)$ & $21(35.6 \%)$ & 0.9030 \\
\hline eGFR ( $\mathrm{ml} \mathrm{min}{ }^{-1}$ per $\left.1.73 \mathrm{~m}^{2}\right)$ & $58.7 \pm 2.8$ & $63.9 \pm 2.9$ & 0.0975 \\
\hline 24-h SBP (mm Hg) & $134.4 \pm 2.2$ & $129.7 \pm 2.2$ & 0.1378 \\
\hline 24-h DBP (mm Hg) & $76.1 \pm 1.6$ & $79.6 \pm 1.5$ & 0.1123 \\
\hline Total cholesterol ( $\left.\mathrm{mg} \mathrm{dl}^{-1}\right)$ & $194.7 \pm 4.6$ & $193.6 \pm 4.6$ & 0.4326 \\
\hline Triglyceride $\left(\mathrm{mg} \mathrm{dl}^{-1}\right)$ & $129.0 \pm 9.9$ & $119.2 \pm 8.4$ & 0.2255 \\
\hline HDL cholesterol $\left(\mathrm{mgdl}^{-1}\right)$ & $54.1 \pm 2.5$ & $57.3 \pm 2.1$ & 0.1648 \\
\hline LDL cholesterol (mg dl-1) & $112.1 \pm 3.8$ & $113.7 \pm 4.1$ & 0.6114 \\
\hline $\mathrm{UA}\left(\mathrm{mgdl}^{-1}\right)$ & $6.1 \pm 0.2$ & $5.7 \pm 0.2$ & 0.2961 \\
\hline $\mathrm{HbA1c}(\%)$ & $5.9 \pm 0.2$ & $5.8 \pm 0.1$ & 0.2330 \\
\hline Macroalbuminuria & $18(37.5 \%)$ & $14(30.4 \%)$ & 0.4699 \\
\hline Mean IMT (mm) & $0.93 \pm 0.04$ & $0.88 \pm 0.04$ & 0.1532 \\
\hline Plaque score & $6.73 \pm 1.05$ & $5.37 \pm 0.92$ & 0.1655 \\
\hline Resistive index & $0.71 \pm 0.01$ & $0.68 \pm 0.01$ & 0.0144 \\
\hline
\end{tabular}

Values are expressed as mean \pm s.e.m. or numbers.

Abbreviations: AASI, ambulatory arterial stiffness index; ABPM, ambulatory blood pressure monitoring; BMI, body mass index; $\mathrm{CCB}$, calcium channel blocker; eGFR, glomerular filtration rate; $\mathrm{HbA1c}$, hemoglobin $\mathrm{Alc}$; $\mathrm{HDL}$, high-density lipoprotein; IMT, intima-media thickness:

LDL, low-density lipoprotein; UA, uric acid; 24-h DBP, 24-h diastolic blood pressure (all-day, evaluated with ABPM); 24-h SBP, 24-h systolic blood pressure (all-day, evaluated with ABPM).

Table 2 Comparison of baseline characteristics and parameters related to systemic atherosclerotic changes between the subjects with greater SBP s.d. and subjects with lower SBP s.d.

\begin{tabular}{|c|c|c|c|c|c|c|c|c|c|}
\hline & \multicolumn{2}{|c|}{ SBP s.d. (all-day) } & \multirow[b]{2}{*}{ P-value } & \multicolumn{2}{|c|}{ SBP s.d. (daytime) } & \multirow[b]{2}{*}{ P-value } & \multicolumn{2}{|c|}{ SBP s.d. (nighttime) } & \multirow[b]{2}{*}{ P-value } \\
\hline & Higher & Lower & & Higher & Lower & & Higher & Lower & \\
\hline Number & 60 & 60 & & 60 & 60 & & 60 & 60 & \\
\hline Sex (male/female) & $18 / 42$ & $32 / 28$ & 0.0095 & $20 / 40$ & $30 / 30$ & 0.0641 & 25/35 & 25/35 & 1.0000 \\
\hline Age (years) & $70.7 \pm 1.6$ & $66.8 \pm 1.6$ & 0.0426 & $71.3 \pm 1.5$ & $66.2 \pm 1.6$ & 0.0114 & $71.6 \pm 1.4$ & $65.9 \pm 1.7$ & 0.0056 \\
\hline BMI $\left(\mathrm{kgm}^{-2}\right)$ & $24.4 \pm 0.6$ & $23.2 \pm 3.3$ & 0.0650 & $24.5 \pm 0.6$ & $23.1 \pm 0.5$ & 0.0283 & $24.6 \pm 0.5$ & $23.1 \pm 0.5$ & 0.0228 \\
\hline Diabetes mellitus ( $n, \%)$ & $19(35.2 \%)$ & $14(26.4 \%)$ & 0.3260 & $21(40.4 \%)$ & $12(21.8 \%)$ & 0.0377 & $16(29.6 \%)$ & $17(32.1 \%)$ & 0.7842 \\
\hline Dyslipidemia $(n, \%)$ & $27(45.0 \%)$ & $16(27.1 \%)$ & 0.0423 & $27(45.0 \%)$ & $16(27.1 \%)$ & 0.0423 & $25(41.7 \%)$ & $18(30.5 \%)$ & 0.2052 \\
\hline eGFR $\left(\mathrm{ml} \mathrm{min}{ }^{-1}\right.$ per $\left.1.73 \mathrm{~m}^{2}\right)$ & $60.9 \pm 2.7$ & $61.8 \pm 3.0$ & 0.4105 & $58.1 \pm 2.6$ & $64.6 \pm 3.0$ & 0.0534 & $59.4 \pm 2.7$ & $63.2 \pm 2.9$ & 0.1718 \\
\hline 24-h SBP (mm Hg) & $132.8 \pm 2.2$ & $131.3 \pm 2.3$ & 0.6428 & $131.4 \pm 2.2$ & $132.7 \pm 2.3$ & 0.6897 & $132.8 \pm 1.9$ & $131.2 \pm 2.5$ & 0.6216 \\
\hline 24-h DBP (mm Hg) & $76.9 \pm 1.6$ & $78.8 \pm 1.5$ & 0.4102 & $76.2 \pm 1.7$ & $79.5 \pm 1.4$ & 0.1298 & $77.1 \pm 1.2$ & $78.6 \pm 1.9$ & 0.4992 \\
\hline Total cholesterol (mg dl-1) & $194.0 \pm 5.0$ & $194.4 \pm 4.2$ & 0.5274 & $192.5 \pm 4.4$ & $195.9 \pm 4.7$ & 0.6006 & $196.1 \pm 4.8$ & $192.3 \pm 4.4$ & 0.2819 \\
\hline Triglyceride $\left(\mathrm{mg} \mathrm{dl}{ }^{-1}\right)$ & $125.8 \pm 7.0$ & $122.2 \pm 11.2$ & 0.3934 & $125.1 \pm 7.1$ & $123.1 \pm 11.1$ & 0.4387 & $127.4 \pm 8.3$ & $120.4 \pm 10.2$ & 0.2982 \\
\hline HDL cholesterol (mg dl-1) & $54.6 \pm 2.4$ & $56.9 \pm 2.2$ & 0.2488 & $54.4 \pm 2.3$ & $57.0 \pm 2.2$ & 0.2133 & $54.3 \pm 2.1$ & $57.1 \pm 2.5$ & 0.1983 \\
\hline LDL cholesterol (mg dl $\left.{ }^{-1}\right)$ & $112.4 \pm 4.0$ & $113.6 \pm 4.0$ & 0.5891 & $111.4 \pm 3.5$ & $114.6 \pm 4.5$ & 0.7122 & $114.2 \pm 4.2$ & $111.5 \pm 3.7$ & 0.3149 \\
\hline $\mathrm{UA}(\mathrm{mgdl}-1)$ & $5.8 \pm 0.2$ & $6.0 \pm 0.2$ & 0.7912 & $6.0 \pm 0.2$ & $5.7 \pm 0.2$ & 0.3198 & $5.9 \pm 0.2$ & $5.9 \pm 0.2$ & 0.8747 \\
\hline $\mathrm{HbAlc}(\%)$ & $6.0 \pm 0.2$ & $5.6 \pm 0.1$ & 0.0413 & $6.1 \pm 0.2$ & $5.6 \pm 0.1$ & 0.0177 & $5.9 \pm 0.2$ & $5.8 \pm 0.1$ & 0.2615 \\
\hline Albuminuria & $16(34.8 \%)$ & $16(33.3 \%)$ & 0.8822 & $18(36.0 \%)$ & $14(31.88 \%)$ & 0.6694 & $15(31.9 \%)$ & $17(36.2 \%)$ & 0.6633 \\
\hline Mean IMT (mm) & $0.96 \pm 0.04$ & $0.85 \pm 0.03$ & 0.0204 & $0.95 \pm 0.04$ & $0.86 \pm 0.03$ & 0.0538 & $0.98 \pm 0.04$ & $0.83 \pm 0.03$ & 0.0030 \\
\hline Plaque score & $7.85 \pm 1.13$ & $4.23 \pm 0.72$ & 0.0043 & $7.04 \pm 0.98$ & $5.12 \pm 0.99$ & 0.0863 & $8.09 \pm 1.14$ & $4.17 \pm 0.73$ & 0.0026 \\
\hline Resistive index & $0.72 \pm 0.01$ & $0.68 \pm 0.01$ & 0.0013 & $0.73 \pm 0.01$ & $0.67 \pm 0.01$ & $<0.0001$ & $0.72 \pm 0.01$ & $0.68 \pm 0.01$ & 0.0057 \\
\hline
\end{tabular}

SBP s.d. was analyzed separately as all-day s.d., daytime s.d. and nighttime s.d. Values are expressed as mean \pm s.e.m. or numbers.

Abbreviations: ABPM, ambulatory blood pressure monitoring; BMI, body mass index; CCB, calcium channel blocker; eGFR, glomerular filtration rate; HbA1c, hemoglobin A1c; HDL, high-density lipoprotein; IMT, intima-media thickness; LDL, low-density lipoprotein; UA, uric acid; 24-h DBP, 24-h diastolic blood pressure (all-day, evaluated with ABPM); 24-h SBP, 24-h systolic blood pressure (all-day, evaluated with ABPM). 


\section{eGFR}

a

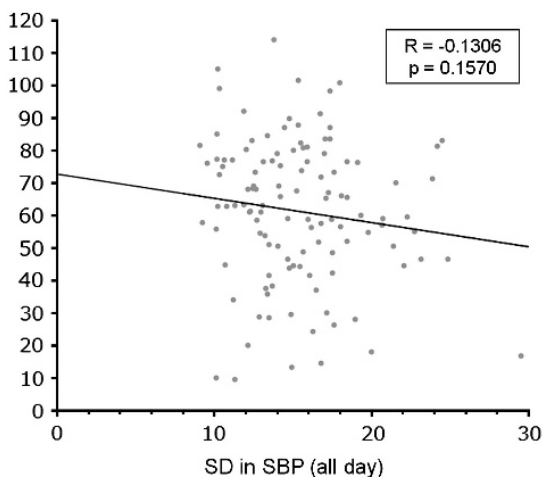

c

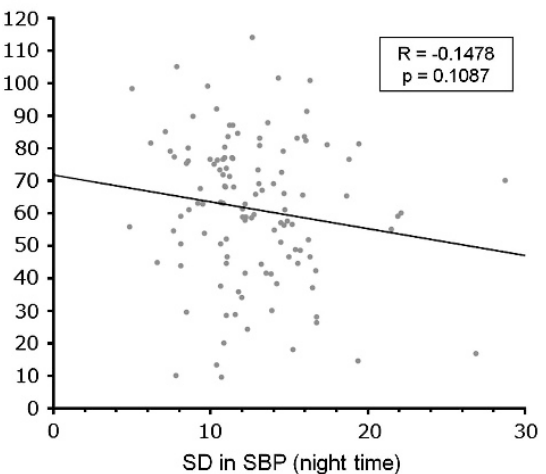

b

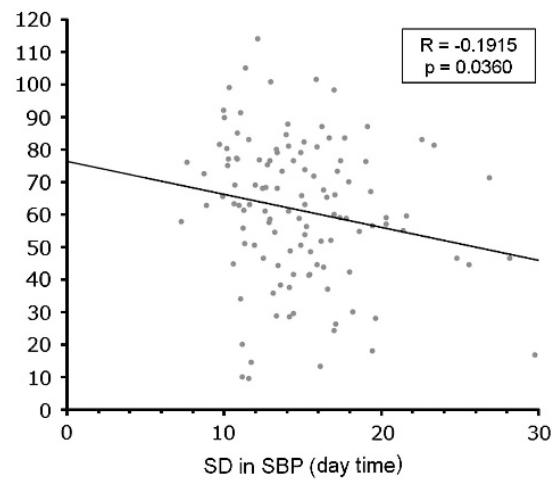

d

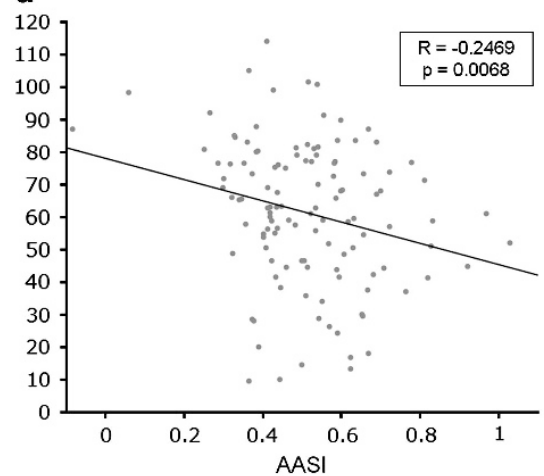

Figure 1 (a) A scatter plot and regression graph of systolic blood pressure (SBP) s.d. (all-day) and estimated glomerular filtration rate (eGFR). There were no significant correlations between SBP s.d. (all-day) and eGFR $(R=-0.1306, P=0.1570)$. (b) A scatter plot and regression graph of SBP s.d. (daytime) and eGFR. eGFR was significantly correlated with SBP s.d. (daytime) $(R=-0.1915, P=0.0360)$. (c) A scatter plot and regression graph of SBP s.d. (nighttime) and eGFR. There were no significant correlations between SBP s.d. (nighttime) and eGFR $(R=-0.1478, P=0.1087)$. (d) A scatter plot and regression graph of ambulatory arterial stiffness index (AASI) and eGFR. eGFR was significantly correlated with AASI $(R=-0.2469, P=0.0068)$.

between subjects with higher and lower all-day s.d. in SBP, daytime s.d. in SBP, nighttime s.d. in SBP and AASI.

Figure 1 shows the correlation between SBP s.d. or AASI and eGFR. eGFR was significantly correlated with daytime SBP s.d. $(R=-0.1915$, $P=0.0360)$ and AASI $(R=-0.2469, P=0.0068)$. On the other hand, there was no significant correlation between eGFR and allday SBP s.d. $(R=-0.1306, P=0.1570)$ and nighttime s.d. in SBP $(R=-0.1478, P=0.1087)$. Figure 2 shows the correlation between SBP s.d. or AASI and RI. RI was significantly correlated with all-day SBP s.d. $(R=0.3122, P=0.0005)$, daytime SBP s.d. $(R=0.3610$, $P<0.0001)$, nighttime SBP s.d. $(R=0.2833, P=0.0017)$ and AASI $(R=0.3149, P=0.0005)$. Figure 3 shows the correlation between s.d. in SBP or AASI and age. Age was significantly correlated with nighttime SBP s.d. $(R=0.2395, P=0.0084)$ and AASI $(R=0.1851$, $P=0.0429)$. Meanwhile, there was no significant correlation between age and all-day SBP s.d. $(R=0.1554, P=0.0902)$ and daytime SBP s.d. $(R=0.1333, P=0.1468)$.

Finally, using multiple regression analysis, we analyzed how SBP s.d. and AASI affect RI. Without adjustment, all-day SBP s.d., daytime SBP s.d., nighttime SBP s.d. and AASI were significantly correlated with RI $(P=0.0005, P<0.0001, P=0.0017$ and $P=0.0005$, respectively). In model 1 , which was adjusted for traditional risk factors (age, sex, BMI, 24-h SBP (evaluated with ABPM)) and serum creatinine level, all-day SBP s.d., daytime SBP s.d. and AASI were significantly and independently correlated with RI $(P=0.0041$,
$P=0.0003$ and $P=0.0290$, respectively). However, nighttime SBP s.d. was not significantly correlated with RI $(P=0.0717)$. In model 2 , which was adjusted for model 1 and with or without diabetes mellitus, dyslipidemia and proteinuria, all-day SBP s.d., daytime SBP s.d. and AASI were also significantly and independently correlated with RI $(P=0.0120, P=0.0052$ and $P=0.0106$, respectively). Meanwhile, nighttime SBP s.d. was not significantly correlated with RI $(P=0.0879)$ (Table 4$)$.

To further investigation, we conducted similar analyses using coefficient variant (CV) and obtained similar results. Subjects with higher SBP CV (all-day, daytime, nighttime) showed significantly higher age $(P=0.0138, P<0.0001$ and $P=0.0017$, respectively). Subjects with higher daytime SBP CV showed significantly lower eGFR (56.4 \pm 2.5 vs. $66.4 \pm 3.1, P=0.0062)$, on the other hand, there were no significant correlation between all-day SBP CV/nighttime SBP CV and eGFR ( $P=0.0958$ and $P=0.3260$, respectively). Subjects with higher nighttime SBP CV showed significantly higher mean IMT ( $0.97 \pm 0.04$ vs. $0.84 \pm 0.03, P=0.0101)$ and plaque score $(7.89 \pm 1.11$ vs. $4.19 \pm 0.74, P=0.0036)$, meanwhile there were no significant correlation between all-day SBP CV/daytime SBP CV and mean IMT $(P=0.1049$ and $P=0.1082$, respectively) or plaque score ( $P=0.0783$ and $P=0.1495$, respectively). And subjects with higher SBP CV (all-day, daytime, nighttime) showed significantly higher RI $(0.73 \pm 0.01$ vs. $0.67 \pm 0.01, P=0.0001,0.73 \pm 0.01$ vs. $0.67 \pm 0.01$, $P<0.0001$ and $0.71 \pm 0.01$ vs. $0.68 \pm 0.01, P=0.0224$, respectively). 


\section{RI}
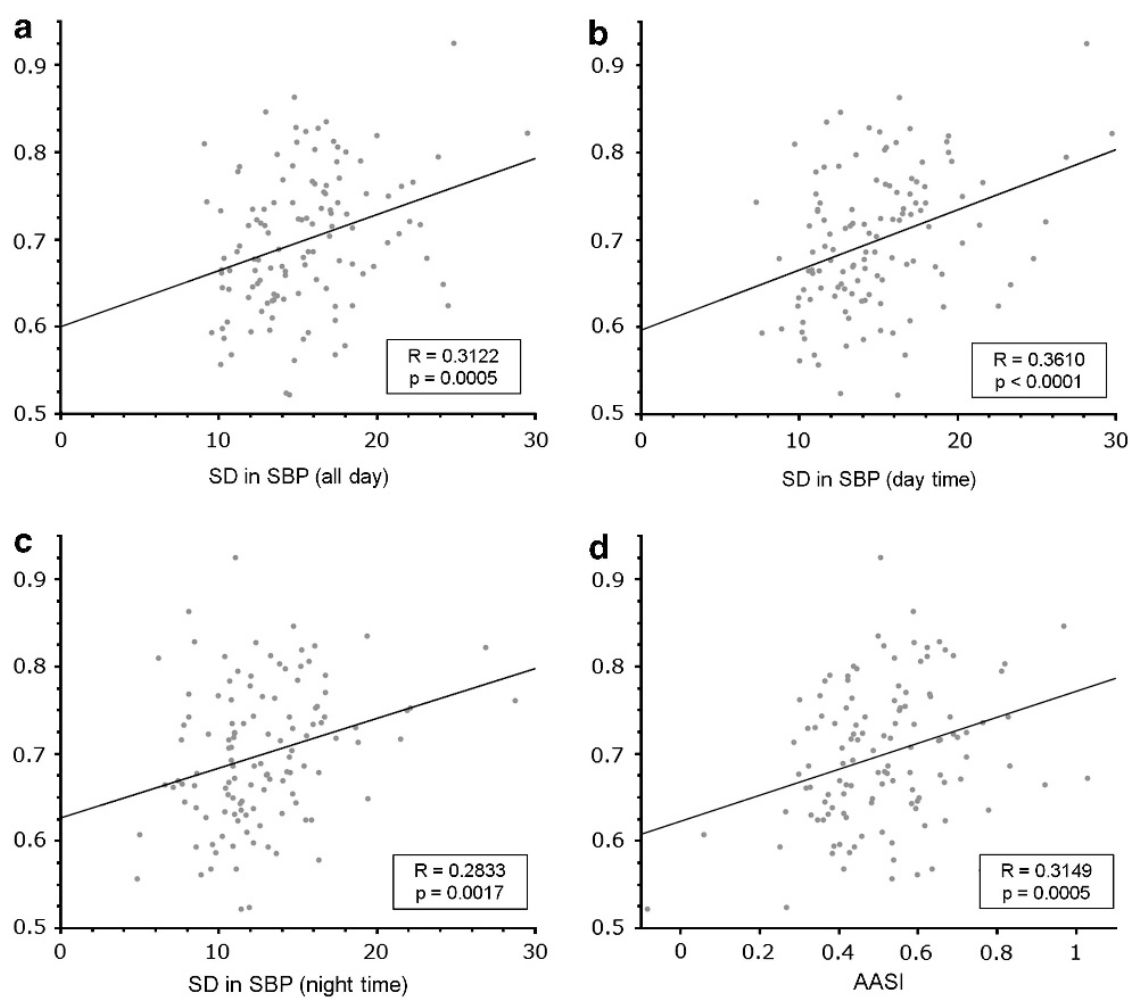

Figure 2 (a) A scatter plot and regression graph of systolic blood pressure (SBP) s.d. (all-day) and resistive index (RI). RI was significantly correlated with SBP s.d. (all-day) $(R=0.3122, P=0.0005)$. (b) A scatter plot and regression graph of s.d. in SBP (daytime) and RI. RI was significantly correlated with SBP s.d. (daytime) $(R=0.3610, P<0.0001)$. (c) A scatter plot and regression graph of SBP s.d. (nighttime) and RI. RI was significantly correlated with s.d. in SBP (nighttime) $(R=0.2833, P=0.0017)$. (d) A scatter plot and regression graph of AASI and RI. RI was significantly correlated with AASI $(R=0.3149, P=0.0005)$

We also conducted multiple regression analysis. Without adjustment, all-day SBP CV, daytime SBP CV, nighttime SBP CV were significantly correlated with RI $(P=0.0008, P<0.0001, P=0.0017$ and $P=0.0140$, respectively). In model 1 , which was adjusted for traditional risk factors (age, sex, BMI, 24-h SBP (evaluated with ABPM)) and serum creatinine level, all-day SBP CV, daytime SBP CV were significantly and independently correlated with RI $(P=0.0042, P=0.0003$, respectively). However, nighttime SBP CV was not significantly correlated with RI $(P=0.1785)$. In model 2 , which was adjusted for model 1 and with or without diabetes mellitus, dyslipidemia and proteinuria, allday SBP CV, daytime SBP CV were also significantly and independently correlated with RI $(P=0.0094, P=0.0057$, respectively). Meanwhile, nighttime SBP CV was not significantly correlated with RI $(P=0.1353)$.

\section{DISCUSSION}

In the present study, we report the correlation between the SBP s.d. or AASI and various parameters related to systemic atherosclerotic change including RI, IMT and plaque score. Higher circadian variability in SBP was significantly correlated with older age and BMI. Our study has several novelties. First, although RI was significantly correlated with all-day SBP s.d., daytime SBP s.d., nighttime SBP s.d. and AASI, we found that daytime SBP s.d. correlated more strongly with RI than other variabilities in SBP. Similarly, daytime SBP s.d. was significantly correlated with the morbidities of diabetes mellitus and elevated HbAlc, but nighttime SBP s.d. showed no significant correlation with HbAlc. Meanwhile, we also showed that nighttime SBP s.d. was significantly correlated with IMT, and plaque score, but daytime SBP s.d. showed no significant correlation with these parameters. These results are consistent with a previous report showing that nighttime BP variability could predict cardiovascular events in patients with type 2 diabetes, ${ }^{18}$ as IMT and plaque score are well known to be strongly associated with stroke. ${ }^{37-39}$ Moreover, in Figure 3, the univariate analysis showed that nighttime SBP s.d., but not daytime SBP s.d., was significantly correlated with age.

Although the reason why daytime variability in BP and nighttime $\mathrm{BP}$ variability showed different impacts on clinical parameters related to atherosclerotic change is unclear. In addition to evidence showing that circadian BP variability is an early sign of autonomic dysfunction, ${ }^{40}$ Eguch et al. ${ }^{18}$ suggested that increased nighttime variability in SBP might be modulated by impaired parasympathetic activity. In contrast, Narkiewicz et al. ${ }^{41}$ suggested that increased daytime BP variability is accompanied by increased sympathetic activity. Based on these results, we thought that daytime BP variability would correlate more strongly with RI and eGFR than nighttime BP variability because the sympathetic overdrive was reported to parallel the severity of renal failure, ${ }^{42}$ and daytime BP variability was accompanied by increased sympathetic activity. Similarly, we thought that nighttime variability, not daytime variability in $\mathrm{BP}$ could be related to age and carotid atherosclerosis because decreased parasympathetic activity was reported to be associated with aging and carotid atherosclerosis 


\section{age}
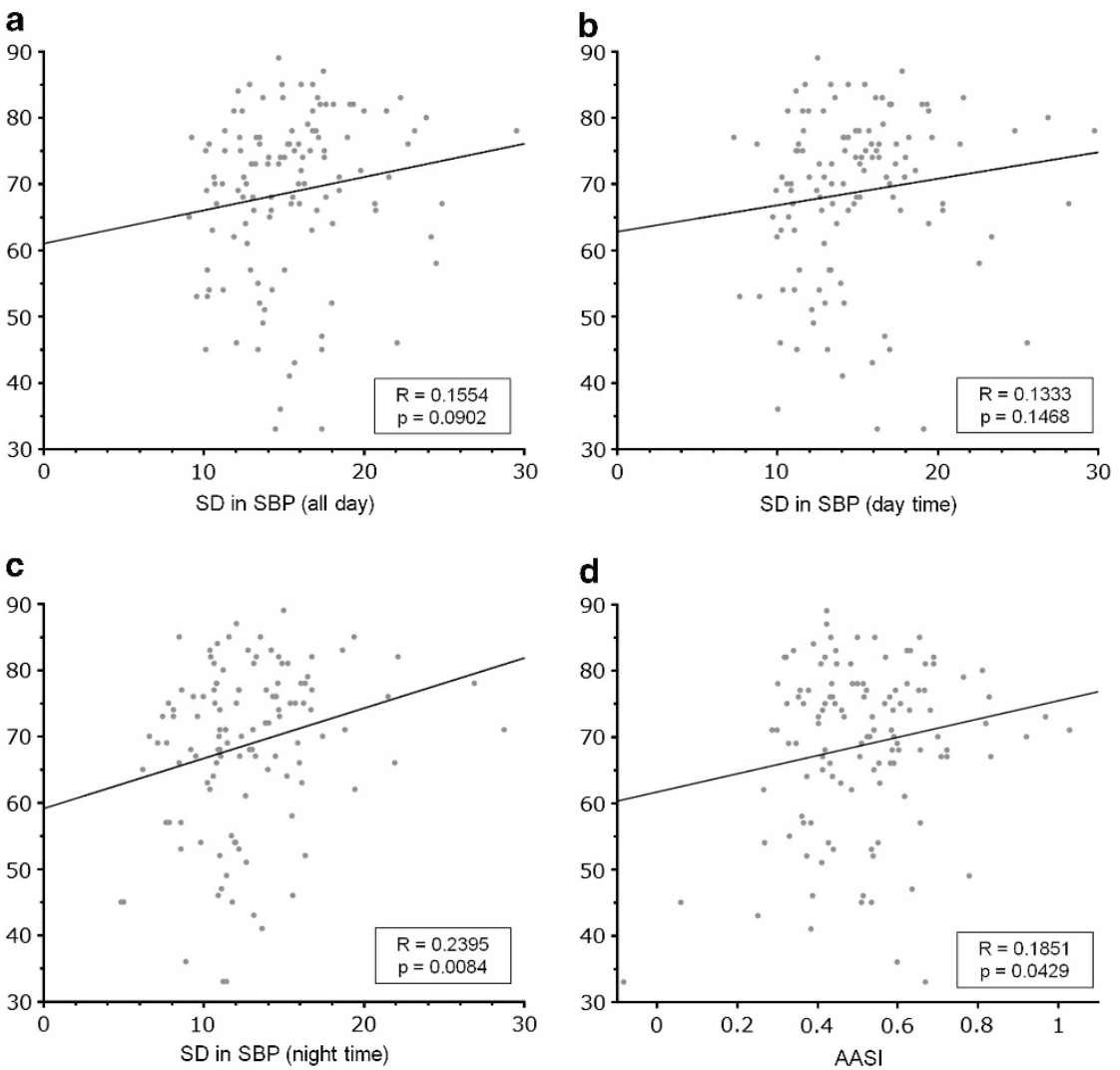

Figure 3 (a) A scatter plot and regression graph of systolic blood pressure (SBP) s.d. (all-day) and age. There were no significant correlations between SBP s.d. (all-day) and age $(R=0.1554, P=0.0902)$. (b) A scatter plot and regression graph of SBP s.d. (daytime) and age. There were no significant correlations between SBP s.d. (daytime) and age $(R=0.1333, P=0.1468)$. (c) A scatter plot and regression graph of s.d. in SBP (nighttime) and age. Age was significantly correlated with SBP s.d. (nighttime) $(R=0.2395, P=0.0084)$. (d) A scatter plot and regression graph of AASI and age. Age was significantly correlated with AASI $(R=0.1851, P=0.0429)$.

Table 4 Correlation between SBP s.d./AASI and RI

\begin{tabular}{lrrrr}
\hline & \multicolumn{4}{c}{ P-value } \\
\cline { 2 - 5 } & $\begin{array}{r}\text { s.d. in SBP } \\
\text { (all-day) }\end{array}$ & $\begin{array}{r}\text { s.d. in SBP } \\
\text { (daytime) }\end{array}$ & $\begin{array}{r}\text { s.d. in SBP } \\
\text { (nighttime) }\end{array}$ & AASI \\
\hline Unadjusted & 0.0005 & $<0.0001$ & 0.0017 & 0.0005 \\
Model 1 & 0.0041 & 0.0003 & 0.0717 & 0.0290 \\
Model 2 & & & & \\
Adjusted for model & 0.0120 & 0.0052 & 0.0879 & 0.0106 \\
$1+$ with or without & & & & \\
DM/ dyslipidemia/ albuminuria & & & & \\
\hline
\end{tabular}

Abbreviations: AASI, ambulatory arterial stiffness index; DM, diabetes mellitus; RI, resistive index; SBP, systolic blood pressure.

Unadjusted model, model 1 (adjusted for age, sex, body mass index, 24-h SBP (evaluated with ambulatory blood pressure monitoring), serum creatinine level), model 2 (adjusted for model

$1+$ with or without diabetes mellitus, dyslipidemia and albuminuria) are compared.

independent of established risk factors. ${ }^{43}$ Also, nighttime BP variability was accompanied by decreased parasympathetic activity. Recently, weighted s.d. in BP, which could exclude the confounding contribution of BP reduction between day and night, was found to be a convenient and useful index to correlate with end-organ damage. ${ }^{44}$
However, the results of the present study indicate the clinical usefulness of using both daytime and nighttime variability in BP, not just all-day variability or weighted variability, to evaluate the pathological conditions of hypertensive patients to prevent cardiovascular diseases.

The second novelty of our study is that we used RI assessed by renal Doppler ultrasonography in all subjects. RI is a valuable index for evaluating renal vascular resistance, and RI reportedly serves as a useful marker to detect and evaluate atherosclerotic diseases due to cardiovascular risk factors, such as hypertension, diabetes mellitus, dyslipidemia and metabolic syndrome. ${ }^{24-26}$ RI also provides good prognostic information for renal function. ${ }^{27-29}$ Moreover, RI was significantly and independently correlated with histopathological atherosclerotic changes analyzed by renal biopsy. ${ }^{45}$ In this study, circadian BP variability correlated more strongly with RI than eGFR. As eGFR is calculated easily from serum creatinine level, and age and sex are useful for renal function screening, it is generally difficult to assess the pathogenesis of CKD using only eGFR. RI could more accurately evaluate very early renal vascular damage than eGFR. ${ }^{10}$ The present study, using RI to assess renal function and pathological change, could evaluate the clinical impact of BP variability and AASI on the kidney more precisely. We found that several variabilities in BP were significantly and independently correlated with RI, even after adjusting for traditional risk factors and serum creatinine level or 
albuminuria, showing that using BP variability in clinical practice, in addition to the traditional evaluation index of renal function, is useful.

The present study has several limitations. First, as this study was an observational, cross-sectional study and the sample size was relatively small, larger longitudinal prospective studies are needed to evaluate the usefulness of circadian variability and AASI in predicting the progression of systemic atherosclerotic change and renal prognosis. Second, as this study was conducted at a university hospital and the study subjects were recruited there, many study subjects had already taken various orally administered drugs for hypertension, dyslipidemia and diabetes mellitus at the time of the investigation, and these agents might have affected several parameters, such as BP, lipid profile and so on. Third, as all of our subjects underwent ABPM in hospitalized condition, it is not clear that the diurnal variation, we evaluated in this study could be equal to diurnal variation in daily life, although ABPM in hospitalized condition enable us to evaluate the diurnal variation without being influenced much by external factors, such as change in temperature or stress about work. Finally, more frequent $\mathrm{BP}$ measurement at night (for example, 15- to 30-min interval) might reveal more accurate correlation between nighttime variability and various parameters even though some of previous studies adopted 60-min interval to evaluate nighttime BP in ABPM and showed meaningful results, ${ }^{10,46}$ so further investigation is required about these points.

In conclusion, circadian SBP variability and AASI were both significantly correlated with renal function; daytime SBP s.d. was especially more strongly correlated with renal vascular resistance, and nighttime SBP s.d. was significantly correlated with IMT and plaque score. These results indicate that calculating both daytime and nighttime BP variability enable an evaluation of the pathological condition of hypertensive patients to prevent cardiovascular diseases.

1 Shulman NB, Ford CE, Hall WD, Blaufox MD, Simon D, Langford HG, Schneider KA Prognostic value of serum creatinine and effect of treatment of hypertension on renal function. Results from the hypertension detection and follow-up program. The hypertension detection and follow-up program cooperative group. Hypertension 1989: 13(5 Supp|): 180-193.

2 Walker WG, Neaton JD, Cutler JA, Neuwirth R, Cohen JD. Renal function change in hypertensive members of the Multiple Risk Factor Intervention Trial. Racial and treatment effects. The MRFIT Research Group. JAMA 1992; 268 3085-3091.

3 Chavanu K, Merkel J, Quan AM. Role of ambulatory blood pressure monitoring in the management of hypertension. Am J Health Syst Pharm 2008; 65: 209-218.

4 Clement DL, De Buyzere ML, De Bacquer DA, de Leeuw PW, Duprez DA, Fagard RH, Gheeraert PJ, Missault LH, Braun JJ, Six RO, Van Der Niepen P, O'Brien E. Office versus ambulatory pressure study investigators: prognostic value of ambulatory bloodpressure recordings in patients with treated hypertension. N Engl J Med 2003; 348 2407-2415.

5 Lewington S, Clarke R, Qizilbash N, Peto R, Collins R. Age-specific relevance of usua blood pressure to vascular mortality: a meta-analysis of individual data for one million adults in 61 prospective studies. Lancet 2002; 360: 1903-1913.

6 Dolan E, Stanton A, Thijs L, Hinedi K, Atkins N, McClory S, Den Hond E, McCormack $P$, Staessen JA, O'Brien E. Superiority of ambulatory over clinic blood pressure measurement in predicting mortality: the Dublin outcome study. Hypertension 2005; 46: $156-161$

7 Verdecchia P, Schillaci G, Guerrieri M, Gatteschi C, Benemio G, Boldrini F, Porcellati C. Circadian blood pressure changes and left ventricular hypertrophy in essentia hypertension. Circulation 1990; 81: 528-536.

8 Ohkubo T, Imai Y, Tsuji I, Nagai K, Watanabe N, Minami N, Kato J, Kikuchi N, Nishiyama A, Aihara A, Sekino M, Satoh H, Hisamichi S. Relation between nocturnal decline in blood pressure and mortality. The Ohasama Study. Am J Hypertens 1997 10: 1201-1207.

9 Timio M, Venanzi S, Lolli S, Lippi G, Verdura C, Monarca C, Guerrini E. 'Non-dipper' hypertensive patients and progressive renal insufficiency: a 3-year longitudinal study. Clin Nephrol 1995; 43: 382-387.

10 Kawai T, Kamide K, Onishi M, Yamamoto-Hanasaki H, Baba Y, Hongyo K, Shimaoka I, Tatara Y, Takeya Y, Ohishi M, Rakugi H. Usefulness of the resistive index in renal
Doppler ultrasonography as an indicator of vascular damage in patients with risks of atherosclerosis. Nephrol Dial Transplant 2011; 26: 3256-3262.

11 Sander D, Kukla C, Klingelhofer J, Winbeck K, Conrad B. Relationship between circadian blood pressure patterns and progression of early carotid atherosclerosis: a 3-year follow-up study. Circulation 2000; 102: 1536-1541.

12 Shintani $Y$, Kikuya M, Hara A, Ohkubo T, Metoki H, Asayama K, Inoue R, Obara T, Aono Y, Hashimoto T, Hashimoto J, Totsune K, Hoshi H, Satoh H, Imai Y. Ambulatory blood pressure, blood pressure variability and the prevalence of carotid artery alteration: the Ohasama study. J Hypertens 2007; 25: 1704-1710.

13 Kikuya M, Hozawa A, Ohokubo T, Tsuji I, Michimata M, Matsubara M, Ota M, Nagai K, Araki T, Satoh H, Ito S, Hisamichi S, Imai Y. Prognostic significance of blood pressure and heart rate variabilities: the Ohasama study. Hypertension 2000; 36: 901-906.

14 Pringle E, Phillips C, Thijs L, Davidson C, Staessen JA, de Leeuw PW, Jaaskivi M, Nachev C, Parati G, O'Brien ET, Tuomilehto J, Webster J, Bulpitt CJ, Fagard RH; SystEur investigators. Systolic blood pressure variability as a risk factor for stroke and cardiovascular mortality in the elderly hypertensive population. J Hypertens $2003 ; 21$ : 2251-2257.

15 Imai Y, Aihara A, Ohkubo T, Nagai K, Tsuji I, Minami N, Satoh H, Hisamichi S. Factors that affect blood pressure variability. A community-based study in Ohasama, Japan. Am J Hypertens 1997; 10: 1281-1289.

16 Hansen TW, Thijs L, Li Y, Boggia J, Kikuya M, Björklund-Bodegård K, Richart T, Ohkubo T, Jeppesen J, Torp-Pedersen C, Dolan E, Kuznetsova T, Stolarz-Skrzypek K Tikhonoff V, Malyutina S, Casiglia E, Nikitin Y, Lind L, Sandoya E, Kawecka-Jaszcz K, Imai Y, Wang J, Ibsen H, O'Brien E, Staessen JA; International Database on Ambulatory Blood Pressure in Relation to Cardiovascular Outcomes Investigators. Prognostic value of reading-to-reading blood pressure variability over 24 hours in 8938 subjects from 11 populations. Hypertension 2010; 55: 1049-1057.

17 Tatasciore A, Renda G, Zimarino M, Soccio M, Bilo G, Parati G, Schillaci G, De Caterina R. Awake systolic blood pressure variability correlates with target-organ damage in hypertensive subjects. Hypertension 2007; 50: 325-332.

18 Eguchi K, Ishikawa J, Hoshide S, Pickering TG, Schwartz JE, Shimada K, Kario K. Night time blood pressure variability is a strong predictor for cardiovascular events in patients with type 2 diabetes. Am J Hypertens 2009; 22: 46-51.

19 Li Y, Wang JG, Dolan E, Gao PJ, Guo HF, Nawrot T, Stanton AV, Zhu DL, O'Brien E, Staessen JA. Ambulatory arterial stiffness index derived from 24-hour ambulatory blood pressure monitoring. Hypertension 2006; 47: 359-364.

20 Dolan E, Thijs L, Li Y, Atkins N, McCormack P, McClory S, O'Brien E, Staessen JA, Stanton AV. Ambulatory arterial stiffness index as a predictor of cardiovascular mortality in the Dublin Outcome Study. Hypertension 2006; 47: 365-370.

21 Leoncini G, Ratto E, Viazzi F, Vaccaro V, Parodi A, Falqui V, Conti N, Tomolillo C, Deferrari G, Pontremoli R. Increased ambulatory arterial stiffness index is associated with target organ damage in primary hypertension. Hypertension 2006; 48: 397-403.

22 Ratto E, Leoncini G, Viazzi F, Vaccaro V, Falqui V, Parodi A, Conti N, Tomolillo C, Deferrari G, Pontremoli R. Ambulatory arterial stiffness index and renal abnormalities in primary hypertension. J Hypertens 2006; 24: 2033-2038.

23 Mulè G, Cottone S, Cusimano P, Incalcaterra F, Giandalia M, Costanzo M, Nardi E, Palermo A, Geraci C, Costa R, Cerasola G. Inverse relationship between ambulatory arterial stiffness index and glomerular filtration rate in arterial hypertension. Am J Hypertens 2008; 21: 35-40.

24 Buscemi S, Verga S, Batsis JA, Cottone S, Mattina A, Re A, Arnone M, Citarda S, Cerasola G. Intra-renal hemodynamics and carotid intima-media thickness in the metabolic syndrome. Diabetes Res Clin Pract 2009; 86: 177-185.

25 Florczak E, Januszewicz M, Januszewicz A, Prejbisz A, Kaczmarska M, Michalowska I, Kabat M, Rywik T, Rynkun D, Zielinski T, Kusmierczyk-Droszcz B, Pregowska-Chwala $B$, Kowalewski G, Hoffman P. Relationship between renal resistive index and early target organ damage in patients with never-treated essential hypertension. Blood Press 2009; 18: 55-61.

26 Raff U, Schmidt BM, Schwab J, Schwarz TK, Achenbach S, Bar I, Schmieder RE. Renal resistive index in addition to low-grade albuminuria complements screening for target organ damage in therapy-resistant hypertension. J Hypertens 2010; 28: $608-614$

27 Fukuda M, Munemura M, Usami T, Nakao N, Takeuchi O, Kamiya Y, Yoshida A, Kimura G. Nocturnal blood pressure is elevated with natriuresis and proteinuria as renal function deteriorates in nephropathy. Kidney Int 2004; 65: 621-625.

28 Platt JF, Rubin JM, Ellis JH. Lupus nephritis: predictive value of conventiona and Doppler US and comparison with serologic and biopsy parameters. Radiology 1997; 203: 82-86.

29 Radermacher J, Ellis S, Haller $\mathrm{H}$. Renal resistance index and progression of rena disease. Hypertension 2002; 39(2 Part 2): 699-703.

30 Schwaighofer B, Kainberger F, Fruehwald F, Huebsch P, Gritzmann N, Karnel F, Tscholakoff D. Duplex sonography of normal renal allografts. Acta Radiol 1989; 30: 53-56.

31 London NJ, Aldoori MI, Lodge VG, Bates JA, Irving HC, Giles GR. Reproducibility of Doppler ultrasound measurement of resistance index in renal allografts. $\mathrm{Br} J$ Radio 1993; 66: 510-513.

32 Knapp R, Plötzeneder A, Frauscher F, Helweg G, Judmaier W, zur Nedden D, Recheis W, Bartsch G. Variability of Doppler parameters in the healthy kidney: an anatomicphysiologic correlation. J Ultrasound Med 1995; 14: 427-429.

33 Mikkonen RH, Kreula JM, Virkkunen PJ. Reproducibility of Doppler ultrasound measurements. Acta Radiol 1996; 37: 545-550.

34 Sakaguchi M, Kitagawa K, Nagai Y, Yamagami H, Kondo K, Matsushita K, Oku N, Hougaku H, Ohtsuki T, Masuyama T, Matsumoto M, Hori M. Equivalence of plaque 
score and intima-media thickness of carotid ultrasonography for predicting severe coronary artery lesion. Ultrasound Med Biol 2003; 29: 367-371.

35 Matsuo S, Imai E, Horio M, Yasuda Y, Tomita K, Nitta K, Yamagata K, Tomino Y, Yokoyama $\mathrm{H}$, Hishida A. Revised equations for estimated GFR from serum creatinine in Japan. Am J Kidney Dis 2009; 53: 982-992.

36 American Diabetes Association Clinical Practice Recommendations 2001. Diabetes Care 2001; 24(Suppl 1): S1-S133.

37 O'Leary DH, Polak JF, Kronmal RA, Manolio TA, Burke GL, Wolfson Jr SK. Carotidartery intima and media thickness as a risk factor for myocardial infarction and stroke in older adults. Cardiovascular Health Study Collaborative Research Group. N Eng/ J Med 1999; 340: 14-22.

38 Lorenz MW, Markus HS, Bots ML, Rosvall M, Sitzer M. Prediction of clinical cardiovascular events with carotid intima-media thickness: a systematic review and meta-analysis. Circulation 2007; 115: 459-467.

39 Shrestha I, Takahashi T, Nomura E, Ohtsuki T, Ohshita T, Ueno H, Kohriyama T, Matsumoto M. Association between central systolic blood pressure, white matter lesions in cerebral MRI and carotid atherosclerosis. Hypertens Res 2009; 32 : 869-874.

40 McKinlay S, Foster C, Clark A, Clark S, Kemp F, Denver E, Coats AJ. Increased blood pressure variability during $24 \mathrm{~h}$ blood pressure monitoring as an early sign of autonomic dysfunction in non-insulin-dependent diabetics. J Hum Hypertens 1994; 8: 887-890.

41 Narkiewicz K, Winnicki M, Schroeder K, Phillips BG, Kato M, Cwalina E, Somers VK. Relationship between muscle sympathetic nerve activity and diurnal blood pressure profile. Hypertension 2002; 39: 168-172.

42 Grassi G, Quarti-Trevano F, Seravalle G, Arenare F, Volpe M, Furiani S, Dell'Oro R, Mancia G. Early sympathetic activation in the initial clinical stages of chronic renal failure. Hypertension 2011; 57: 846-851.

43 Jae SY, Carnethon MR, Heffernan KS, Choi YH, Lee MK, Park WH, Fernhall B. Slow heart rate recovery after exercise is associated with carotid atherosclerosis. Atherosclerosis 2008; 196: 256-261.

44 Bilo G, Giglio A, Styczkiewicz K, Caldara G, Maronati A, Kawecka-Jaszcz K, Mancia G, Parati G. A new method for assessing 24-h blood pressure variability after excluding the contribution of nocturnal blood pressure fall. J Hypertens 2007; 25: 2058-2066.

45 Ikee R, Kobayashi S, Hemmi N, Imakiire T, Kikuchi Y, Moriya H, Suzuki S, Miura S. Correlation between the resistive index by Doppler ultrasound and kidney function and histology. Am J Kidney Dis 2005; 46: 603-609.

46 Furuäng L, Siennicki-Lantz A, Elmståhl S. Reduced cerebral perfusion in elderly men with silent myocardial ischaemia and nocturnal blood pressure dipping. Atherosclerosis 2011; 214: 231-236. 